\title{
The Effect of Fault Tolerance in a WSN Cluster on the QoS
}

\author{
Khushboo Malik ${ }^{1}$, Dr. K. K. Paliwal ${ }^{2}$ \\ Department of Electronics and Communications, Panipat Institute of Engineering and Technology, Panipat
}

Abstract: The principle issue with existing convention lies in the arbitrary choice of cluster heads. There exists a likelihood that the cluster heads structured are unequal and may stay in one part of the system making another part of the system inaccessible. In this work an attempt has been made to enhance the system existence with smart clustering component by dissecting the Connectivity, Distance and Residual proficiencies. The work will additionally decrease the rate of getting a particular node dead, rapidly. The proposed scheme will similarly control the amount of clusters in the system by indicating as far as possible for number of nodes in the cluster. This work will give an attractive balanced circulation of cluster and also sensor nodes over the system.

Keywords: Sensor node, Cluster Head, Maximum Connectivity, Maximum Residual Energy and Maximum Response time

\section{Fault Tolerance}

\section{Introduction}

As the cluster heads $(\mathrm{CH})$ plays a vital role in the functioning of WSN that works in adverse situations with a specific end goal to keep away from the authenticity of paramount sensor's information. Allotting cluster heads is the most striking plan for recovering from a cluster head malfunction. Appointing the cluster heads among live nodes in a cluster can be an efficient method for tolerating the faults in a WSN [5].

A. Increased connectivity and reduced delay time- In WSNs, where the cluster heads are picked from sensor nodes, restricting the extent of integration and up gradation between cluster heads network may be more suitable than a network without a $\mathrm{CH}$. Also, when information idleness is a major concern, intra cluster integration turns into a configuration target or requirement.

B. Maximal network longevity- Since sensor nodes are battery oriented, the system's lifetime is a significant concern particularly for requisitions of WSNs put in harsh situations. Versatile clustering is an important decision with a specific end goal to attain more system life span [19].

\section{2 .Sensor Networks}

Sensor Networks are utilized as a part of an extensive variety of requisitions to catch, accumulate and process live ecological information. A Sensor Network regularly comprises of a Base Station (BS) and a group of sensor nodes. The sensor nodes are in charge of constantly examining physical phenomena, for example, temperature and humidity. They are additionally equipped for communicating with each another and the base station through radios. The base station, serves as a door for the sensor system to exchange information with provisions to fulfill their missions. While the base station can have continuous power supply, the sensor nodes are typically battery-fueled. The batteries are badly designed and once in a while even difficult to be replaced [1].At the point when a sensor node uses up vitality, its scope is lost. The handy estimation of a sensor system is dictated over span before it neglects to complete the mission length of time is alluded to as the system lifetime. It is both mission-discriminating and financially attractive to oversee sensor information in a vitality effective approach to enhance the lifetime of sensor systems.

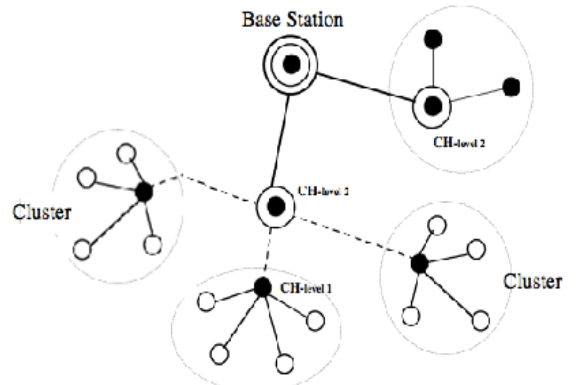

Fig 1: Information forwarding among Sensor nodes with clustering and aggregation 
The information gathered by the sensor nodes are regularly updated into a total structure asked for by the provisions (e.g., normal temperature detection). Fundamentally intended for monitory purposes, numerous sensor requisitions require constant collection in view of the fact that every sensor node need to report each reading to the base station [18].

\section{Clustered Architecture}

The final outcome of any directing convention is to make the system helpful and productive. A cluster based steering convention consists of sensor nodes where each group of nodes has a $\mathrm{CH}$ or a gateway. Sensed information is sent to the $\mathrm{CH}$ instead of sending it to the BS. $\mathrm{CH}$ performs some aggregation work on information it accepts and sends it to the BS where this information is required.

Various directing conventions have been proposed for WSN. A standout amongst the most well known progressive conventions is Low Energy and Adaptive Clustering Hierarchy (LEACH). This shows noteworthy improvement in the general system vitality over other non-clustering convention. Progressive directing conventions intended to decrease the energy consumption by restricting communication inside the cluster and total information to minimize transmissions to the BS [19].

\section{A. Clustering Objective}

This segment exhibit two primary destinations that are significant to the objectives of this research paper.

a. Maximizing network Life-time- Unlike in cell systems, where portable devices (e.g. telephones) can undoubtedly be revived always after maximum usage of battery capacity, administration control in these systems remains an auxiliary issue. On the other hand, in WSN the battery source is exceptionally constrained. As, the sensor nodes are provided with limited power source; for instance the Berkeley's MICA bits are fueled by two AA basic batteries . The fundamental configuration goals of WSN is to effectively utilize the limited power source. Therefore, clustering plan has been sought to enhance system life-time and help simplify the issue of every node transmitting specifically to BS as in conventional methods like Direct Transmission [22].

b. Load balancing- Load balancing system could be an alternate outline objective of clustering plans. It is required not to overload the cluster heads as this may exhaust their energies faster. Therefore, it is paramount to have even distribution of nodes in each cluster particularly in situations where cluster heads are performing aggregations or other assignments.

\section{Existing Work}

In general, the systems employed for WSNs caters for redundancies and could be connected in different levels of framework segments including processors, memory units, sensing range, information streams, transmission timelines and programming codes. For instance, various repetitive circle cluster architectures (Strike) are proposed, on which a bunched structural planning could be fabricated [9]. On-line failure recovering calculations can permit a quick transformation by either retaining the transfer speed or by using the intrinsic repetition of the requirement. We must consider fault tolerance in an arrangement of imparting communication that cooperates to give some administration to the BS such that the network is survivable. In the case of such framework that may be a bunch of workstations giving web administration to a customer, the customer demands a page and the server machines react by conveying that page. A further case is a feature conveyance framework where a server conveys a feature stream to a customer associated with the system. In both situations a fault may be an administration intrusion because of system part failures or crashing of machine.

Conventional fault tolerance has forced to contemplate the issue of managing administration blackouts on the grounds that come up in a framework. Methods include supplanting the faulty component where it is possible by utilizing a hot standby or a warm standby or actually tolerate some downtime and repair the unit logged off. Existing activity might additionally be rerouted through an alternate access way from the source to its goal by directing calculations. In information transfer systems, fault tolerance has advanced to considering the recuperation of deficiencies such as decrease in the pace or limit of an administration. For example, a telecom switch may reduce the number of calls that might be taken care of if a processor failure is experienced.

Furthermore, because of rerouting, these administration failures may cause an adverse effect on administration quality as well for example, decrease in data transfer capacity or expanded idleness. We see Quality of service as another plan to be taken care of in a proactive manner: In the vicinity of shortcomings, administrations that need a level of asset (e.g., transfer speed) should be ensured to perform properly when there is sufficient of the asset to be imparted, and when it is rare, transaction with the administration supplier will occur to guarantee a more level, yet deliverable level of administration.

The research work is about a clustered system under the restriction of no. of nodes in each cluster. The work will control the congestion over the system. The work will additionally transmit the information of high quality. To give the correspondence to existing nodes the multi hop system has been proposed in this work. 


\section{Simulation Results}

The result refers to the measurement of quality of service during transmission. Figures below show the output of improved clustering approach. Transmission quality of a network is related to no. of rerouting experienced by each packet to avoid faulty nodes and the no. of clusters formed. System which is proposed here gives good output in both the parameters.

Parameters which are used for simulation are:

No. of Nodes $(\mathrm{n})=100$

Optimal Election Probability of a node to become cluster head $(\mathrm{P})=0.2$;

Initial Energy $(\mathrm{EO})=0.5 \mathrm{~J}$;

Energy for Transmission $(\mathrm{ETX})=50 * 0.000000001 \mathrm{~J} / \mathrm{bit}$

For Energy Reception $(E R X)=50 * 0.000000001 \mathrm{~J} / \mathrm{bit}$;

Free Space Energy Loss $(\mathrm{Efs})=10 * 0.000000000001 \mathrm{~J} / \mathrm{bit} / \mathrm{m}^{2}$;

Multi path Energy Loss $(E m p)=0.013 * 0.000000000001 \mathrm{~J} / \mathrm{bit} / \mathrm{m}^{4}$;

Data Aggregation Energy $(E D A)=5 * 0.000000001 \mathrm{~J} / \mathrm{bit} /$ signal;

Maximum no. of Rounds $(\operatorname{rmax})=1000$;

Maximum no. of rerouting per packet transmission $=200$;

Time limits $=0$ to 20 seconds per packet transmission

We have taken all these values and found that there are less dead nodes and more alive nodes in proposed system. Also, the rate of packet transmission is enhanced and due to more alive nodes, cluster formation process is ensured for a long time which tends to increase the life time of wireless sensor network. Here figures are presented which shows the output of modified system. The existing clustering approach is also considered for the purpose of comparison. After simulations, the following results were gathered. Based upon these results, a detailed analysis is presented.

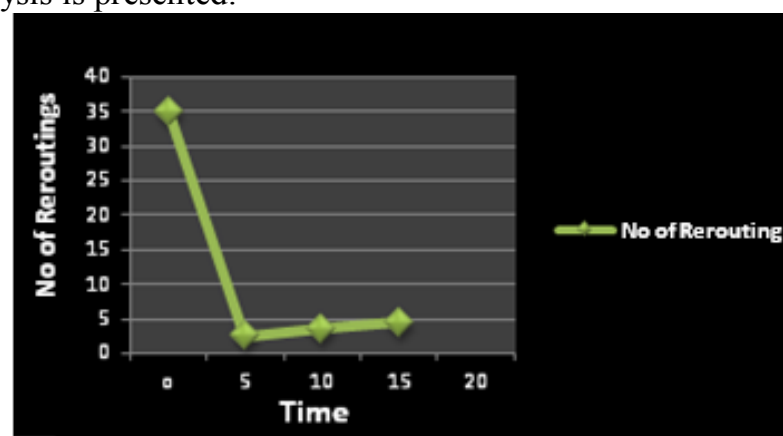

Fig. 2 Transmission quality (Number of rerouting per packet vs. Time in sec) (proposed approach)

Figure 2 shows the analysis of the work in terms of number of rerouting during communication to the base station. As we can see, in proposed approach, the rerouting at the initial stage is high due to high communication over the network. But as the nodes started getting dead, the rate of packet communication decreased and therefore, the no. of rerouting decreased.

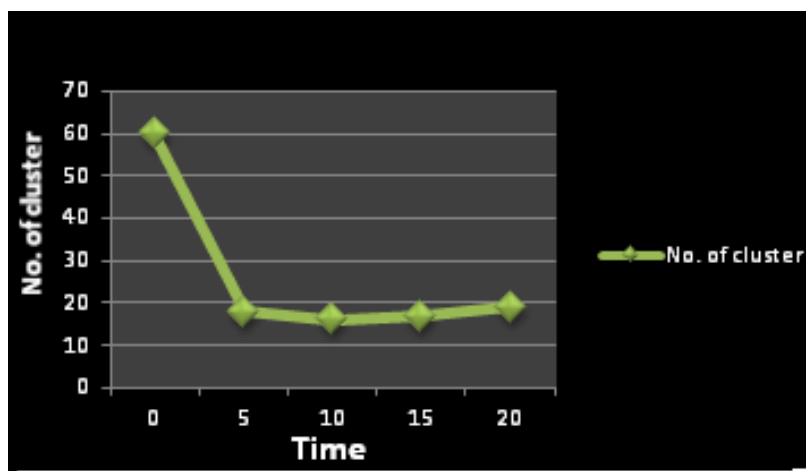

Fig. 3 Inter-cluster Communication (Proposed Approach)

Here in figure 3, the analysis of work is shown in terms of packets transmitted in the network. Transmission is here performed up to 550 rounds of the network. Because of equalized distribution over the network, the inter cluster communication also increased. As the nodes die over the network in equalized mode, 
the inter cluster communication ratio is decreased but more qualitative communication is performed over the network.

\section{Comparative Results-}

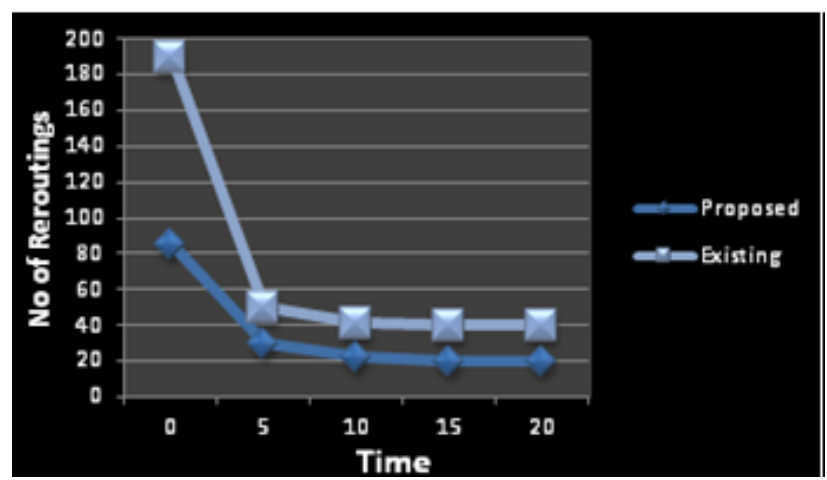

Fig. 4 Packet Transmission quality (Number of rerouting vs. Time in sec) (Existing vs. Proposed)

Figure 4 shows the number of rerouting per packet delivered to the Base Station per second. It is clear from the figure that the number of rerouting per packet delivered by existing approach is more as compared to proposed approach. This concludes that our approach sends more qualitative information to BS for longer period. As the presented approach provided an effective clustering method over the network, the network communication improves, which resulted in the higher transmission of packets over the network and the overall network life also improved.

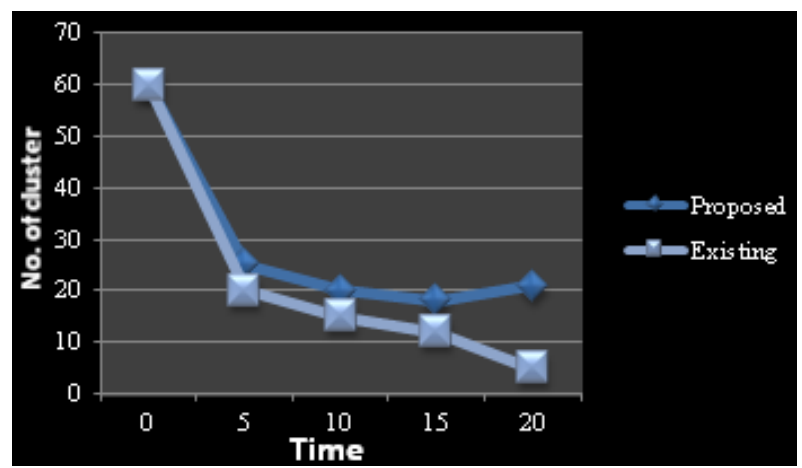

Fig. 5 Inter-cluster Communication (No. of cluster vs. Time) (Existing vs. Proposed)

Figure 5 shows that the higher no. of packets is transmitted in the proposed approach as the network life is increased. The presented approach provided the better clustering method so that the equalized distribution of clusters over the network is performed. The presented work improved the inter cluster communication over the network.

\section{Conclusion}

In this work, we have defined an improved clustering architecture with cluster heads definition. The work is here performed in the cluster head selection procedure. Maximum Connectivity, Maximum Residual Energy and Minimum Response Time will be taken as the choice parameters for the cluster head determination. The proposed framework will enhance the system life as well as the quality of service under faulty circumstances. Simulation results show that the proposed approach enhanced the quality of service as compared to the existing approach.

\section{References}

[1]. Macro conti, Giuseppe Anastasi, Mario Di Francesco, Andrea Passarella, "Energy conservation in wireless sensor networks: A Survey", Ad-hoc network 7 (2009), pp. 537-568.

[2]. S. Venkatesan, Shashidhar Rao ,Gandham. Milind Dawande, Ravi Prakash, "Energy Efficient Scheme for Wireless Sensor Networks with Multiple Mobile Base Stations". Vol-6, IEEE Aug 2003,pp.377-381.

[3]. Miodrag Potkonjak, Sasa Slijepcevic, "Power Efficient Organization of Wireless Sensor Networks". IEEE July 2001, pp.472-476.

[4]. Carlos Carreto, Tiago Camilo, Jorge SA Silva, Fernando Boavida, Energy Efficient Ant-Based Routing Algorithm for Wireless Sensor Network". IEEE 2006, pp 49-59.

[5]. Edward J. Coyle, Seema Bandyopadhyay, Energy Efficient Hierarchical Clustering Algorithm for Wireless Sensor Network". IEEE Sept. 2003, pp. 1713-1723 
[6]. Sach Shelby, Carlos Pomalaza, Heikki Karvonen, Jussi Hapola, "Energy Optimization in Multihop Embedded and Sensor Network". International Journal of Wireless Informatics Network, Vol -12, January 2006, pp. 11-20.

[7]. Neetesh Purohit, Himanshu Aggarwal, Ankit Jain, "A new scheme for Cooperative Communication in LEACH based Wireless Sensor Network”. 2011 International Conference on Computational Intelligence and communication System. pp. 47-51.

[8]. Naeim Rahmani, Heila Kousha, Ladan Darougaran, Farhad Nematy, "CAT : The New Clustering Algorithm based on two-Tier Network Topology for Energy Balancing in Wireless Sensor Networks". 2010 International Conference.

[9]. Mihela Cardei,My T. Thai, Yingshu Li, Weili Wu," Energy Efficient Target Coverage in Wireless Sensor Networks". IEEE 2005, pp. 1976-1984.

[10]. Sundeep Pattem,Alexandre Ciancio, Antonio Ortega and Bhaskar Krishnamachari,"Energy Efficient Data Representation and Routing for WSNs Based on a Distributed Wavelet Compression Algorithm”. IPSN April 2006 pp. 309-316.

[11]. Hyung Seok Kim,Tarek F. Abdelzaher,Wook Hyun Kwon,"Minimum Energy Asynchronous Dissemination to Mobile Sinks in Wireless Sensor Networks". Sensys November 2003 pp. 193-204.

[12]. Jing Li, Georgios Y. Lazarou,"A Bit Map Assisted Energy Efficient MAC Scheme for Wireless Sensor Networks". IPSN April 2004 pp. 55-60.

[13]. Zack Shelby,"UWB Wireless Sensor Networks : UWEN". IEEE December 2004 pp. 27-32.

[14]. Sushant Jain, Rahul C. Shah, Sumit Roy,"Exploiting Mobility For Energy Efficient Data Collection in Wireless Sensor Networks". Springer 2006 pp. 327-339.

[15]. Sudharman K. Jayweera,"Virtual MIMO based Cooperative Communication for Energy constrained Wireless Sensor Networks". IEEE May 2006 pp. 984-989.

[16]. Y. Sankarasubramaniam, I.F. Akyilidiz, S.W. Mc Laughlin,’'Energy Efficient based Packet Size Optimization in Wireless Sensor Networks". IEEE 2003 pp. 1-8.

[17]. Tijs van Dam, Koen Langendoen,"An Adaptive Energy Efficient MAC Protocol for Wireless Sensor Networks". Sensys November 2003 pp. 171-180.

[18]. R. M. Sharma, "Performance Comparison of AODV, DSR and AntHocNet Protocols", Conf. on Mobile computing and networking. pp. 195-206. Sept.1999.

[19]. W. Heinzelman, "Application-specific protocol architectures for wireless networks", Ph.D. thesis, Massachusetts Institute of Technology, 2000.

[20]. Ayad Salhieh, Jennifer Weinmann and Loren Schwiebert, "Power Efficient Topologies for Wireless Sensor Networks" Wireless Networks, vol.5(6),pp.445-460, Augt.1999.

[21]. Xu Geng, Guangsong Yang,"Energy Efficient Route finding Scheme based on Directional Antenna in WSNs". ICISE 2009pp.26252629.

[22]. S.Sudha, N. Hemavathi,"A Hybrid Clustering Algorithm for Optimal Clustering in Wireless Sensor Networks". IEEE 2014. 